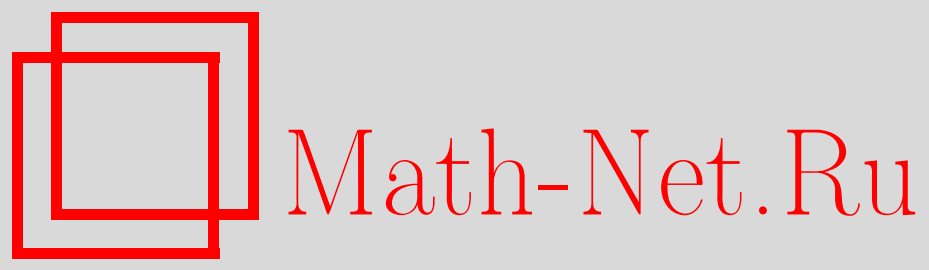

В. А. Зорич, В. М. Кесельман, О конформном типе риманова многообразия, Функи. анализ и его прил., 1996, том 30, выпуск 2, 40-55

DOI: https://doi.org/10.4213/faa520

Использование Общероссийского математического портала MathNet.Ru подразумевает, что вы прочитали и согласны с пользовательским соглашением

http://www . mathnet.ru/rus/agreement

Параметры загрузки:

IP : 3.80 .181 .102

26 апреля 2023 г., 10:11:32

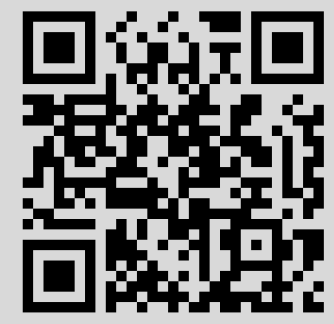


Функииональньй анализ и еәо приложения

1996, т. 30, вып. 2, с. 40-55

УДК $517.54+514.774$

\title{
О конформном типе риманова многообразия
}

\author{
(C) 1996. В. А. ЗОРИЧ ${ }^{1}$, В. М. КЕСЕЛЬМАН
}

Посвящается Ларсу Альфорсу

\section{$\S 1$. Введение}

Понятие конформного типа римановой поверхности, как известно, может быть дано в ее внутренних терминах, без привлечения конформных отображений теории униформизации. Это можно сделать с помощью конформной емкости или других, равносильных ей конформных инвариантов: модуля семейства кривых, экстремальной длины.

В таком виде понятие конформного типа естественно распространяется на римановы, конформные и даже квазиконформные многообразия любой размерности.

А именно, открытое риманово многообразие назовем многообразием конформно-параболического или конформно-гиперболического типа, если конформная емкость его идеальной границы (абсолюта) равна нулю или положительна соответственно.

Ниже (в §2) мы напомним определения упомянутых конформных инвариантов.

Используя свойства конформной емкости и модуля, мы развиваем восходяшие в идейном плане к работам Альфорса $[1,2]$ (см. также $[19 ; 20$, гл. 6] и $[35$, с. 1223$])$ геометрические признаки и критерии конформного типа риманова многообразия, выраженные в терминах изопериметрической функции и интегрального поведения площади геодезических сфер (см. $\S 5,6)$.

Мы отмечаем здесь также (см. §3) следующее ключевое геометрическое наблюдение:

Риманово многообразие является конформно-параболическим тогда и только тогда, когда конформной заменой метрики его можно превратить в полное риманово многообразие конечного объема.

В таком виде оно было сформулировано в докладе [46] и в статье [47, с. 140] и, как будет показано, является геометрической интерпретацией одного свойства модуля [14, с. 179].

Полное исследование поведения объема геодезических шаров в конформном классе метрик риманова многообразия см. в [21].

Интегральные соотношения на римановом многообразии, связанные с проблемой конформного типа, включая лемму Альфорса-Громова, обсуждены в $\S 4$.

${ }^{1}$ Работа выполнена при частичной поддержке РФФИ и AMS. Авторы признательны Н. А. Зорич за подготовку электронной $\mathrm{T}_{\mathrm{E}} \mathrm{X}$-версии рукописи этой статьи. 


\section{§2. Конформные инварианты}

Для удобства читателя напомним определения используемых в дальнейшем конформных инвариантов: конформной емкости и модуля (см., например, [3, 14 , 7, 15-17, 29, 34, 37, 38, 43, 44]).

2.1. Конформная емкость. Пусть $R\left(C_{0}, C_{1}\right)$ - конденсатор на $n$-мерном римановом многообразии $(M, g)$, т. е. область, ограниченная континуумами $C_{0}$, $C_{1}\left(C_{0} \cup C_{1}=\partial R\left(C_{0}, C_{1}\right)\right.$ и $\left.C_{0} \cap C_{1}=\varnothing\right)$. Конформной емкостью конденсатора $R\left(C_{0}, C_{1}\right)$ называется величина

$$
\operatorname{cap} R\left(C_{0}, C_{1}\right):=\inf \int_{M}\|\operatorname{grad} U\|^{n},
$$

где нижняя грань берется по всем вещественнозначным функциям (потенциалам) $U: M \rightarrow \mathbb{R}$ с обобщенными производными, таким, что $\left.U\right|_{C_{0}}=0,\left.U\right|_{C_{1}}=1$.

Например, конформная емкость конденсатора $R_{r_{0}}^{r_{1}} \subset \mathbb{R}^{n}$, ограниченного сферами $C_{0}=\left\{x \in \mathbb{R}^{n}:|x|=r_{0}\right\}, C_{1}=\left\{x \in \mathbb{R}^{n}:|x|=r_{1}\right\}$, где $0<r_{0}<r_{1}$, равна

$$
\text { cap } R_{r_{0}}^{r_{1}}=\omega_{n-1} \ln ^{1-n} r_{1} / r_{0},
$$

где $\omega_{n-1}$ есть $(n-1)$-мера единичной сферы в $\mathbb{R}^{n}$.

2.2. Модуль. Конформная емкость конденсатора может быть описана несколько иначе, в терминах модуля (или экстремальной длины $[4,14])$ семейства кривых. Порой такая форма предпочтительна, хотя все результаты этой работы, использующие емкость и модуль, конечно, можно изложить, ограничившись, например, лишь определением (1) емкости.

Пусть $\Gamma=\{\gamma\}$ - семейство кривых на $n$-мерном римановом многообразии $(M, g)$. Измеримая по Борелю неотрицательная функция $\rho: M \rightarrow \mathbb{R}$ называется допустимой для $\Gamma$, если для любой кривой $\gamma \in \Gamma$

$$
\int_{\gamma} \rho \geqslant 1
$$

Конформным модулем семейства кривых Г называется величина

$$
\bmod (\Gamma):=\inf \int_{M} \rho^{n},
$$

где нижняя грань берется по всем допустимым для $Г$ функциям.

2.3. Взаимосвязь емкости и модуля конденсатора. Если $\Gamma$ - семейство всех кривых в $R\left(C_{0}, C_{1}\right)$, соединяющих граничные компоненты $C_{0}$, $C_{1}$ конденсатора $R\left(C_{0}, C_{1}\right)$, то

$$
\bmod (\Gamma)=\operatorname{cap} R\left(C_{0}, C_{1}\right) .
$$

(Для случая $M=\mathbb{R}^{n}$ доказательство см., например, в $[15,23,45]$. )

Процесс осреднения (свертка с регулярным ядром) позволяет при вычислении емкости конденсатора и модуля соответствующего семейства кривых ограничиться функциями, порядок гладкости которых совпадает с порядком гладкости самого многообразия. 
2.4. Конформная инвариантность емкости и модуля. Локальное изменение в $\lambda$ раз элемента длины на $(M, g)$, т. е. переход к метрике $\lambda^{2} g$, приводит к изменению в $\lambda^{n}$ раз элемента объема и в $\lambda^{-1}$ раз градиента функции; поэтому формула (1) определяет величину, инвариантную относительно конформного изменения метрики.

Аналогично если в соответствии с изменением в $\lambda$ раз элемента длины на $(M, g)$ заменить $\rho$ новой допустимой функцией $\lambda^{-1} \rho$, то можно видеть, что величина (4) - тоже конформный инвариант.

2.5. Конформный тип риманова многообразия. Формула (2) показывает, что сар $R_{a}^{b} \rightarrow 0$ в $\mathbb{R}^{n}$ при $b \rightarrow \infty$ независимо от величины $a$. По этой причине говорят, что емкость пространства $\mathbb{R}^{n}$ в бесконечности (т. е. емкость любого конденсатора $R\left(C_{0}, C_{1}\right)$, когда компонента $C_{1}$ уведена на абсолют) равна нулю. После (конформной) стереографической проекции пространство $\mathbb{R}^{n}$ можно интерпретировать как проколотую сферу $S^{n} \backslash p$, а точку $p \in S^{n}$ — как абсолют.

Метрика Пуанкаре в его модели гиперболического пространства Лобачевского $\mathbb{H}^{n}$ в единичном шаре $B^{n} \subset \mathbb{R}^{n}$ конформно-евклидова. Значит, конформные емкости гиперболических объектов можно вычислять непосредственно в евклидовой метрике. Абсолют пространства $\mathbb{H}^{n}$ совпадает в модели Пуанкаре с граничной сферой $\partial B^{n}$, и в соответствии с формулой (2) сар $R_{a}^{1}>0$ при любом $a \in(0,1)$, т.е. в данном случае емкость абсолюта, или емкость $\mathbb{H}^{n}$ в бесконечности, положительна.

В классической теории римановых поверхностей, когда любая односвязная поверхность конформно отображается на $\mathbb{C} \sim \mathbb{R}^{2}$ или на $\mathbb{H}^{2} \sim B^{2}$, эти два случая различают как параболический и гиперболический конформный тип поверхности соответственно.

В высших размерностях конформных отображений на канонические области принципиально нет (в отличие от двумерного случая и в отличие от просто конформных замен метрики $g$ на $\lambda^{2} g$ ). Рассмотренные конформные инварианты позволяют, однако, дать внутреннее определение конформного типа риманова многообразия, как это указано выше в $\S 1$, не прибегая к конформным отображениям, т.е. оставаясь и проводя измерения только на самом многообразии.

В этой связи ясно, однако, что переход в высшие размерности несколько меняет акценты и содержание понятия конформного типа многообразия.

\section{§3. Конечность объема как критерий конформной параболичности}

В качестве иллюстрации применения рассмотренных конформных инвариантов докажем сформулированный во введении критерий параболичности.

Если открытое риманово многообразие $(M, g)$ полно, то каждая выходящая на абсолют (т.е. не относительно компактная) кривая $\gamma$ имеет бесконечную длину и, значит, любая константа $\varepsilon>0$ является допустимой функцией для семейства $\Gamma_{\infty}$ всех таких кривых. Если многообразие к тому же имеет конечный объем, то ввиду произвольности $\varepsilon$ ясно, что $\bmod \left(\Gamma_{\infty}\right)=0$ и $(M, g)$ конформнопараболично.

Менее очевидно и более интересно обратное утверждение. Пусть $(M, g)$ конформно-параболично. Значит, $\bmod \left(\Gamma_{\infty}\right)=0$, где $\Gamma_{\infty}-$ семейство всех кривых, 
не являющихся относительно компактными на $(M, g)$. По свойству модуля (см. $\left[14\right.$, с. 179]) найдется измеримая функция $\rho$ на $M$, такая, что $\int_{\gamma} \rho=\infty$ на любой кривой $\gamma \in \Gamma_{\infty}$ и в то же время $\int_{M} \rho^{n}<\infty$. Переходя к метрике $\rho^{2} g$ на $M$, получаем полное риманово многообразие конечного объема, что и завершает доказательство.

Например, в $\mathbb{R}^{n}(n>0)$ полная конформно-евклидова метрика $\lambda(x)|d x|$ конечного объема получится, если $\lambda(x) \sim(|x| \ln |x|)^{-1}$ при $|x| \rightarrow+\infty$.

В приведенном доказательстве, чтобы не затемнять его простое содержание, мы позволили себе опустить некоторые детали, на которых теперь остановимся.

Формально рассмотрения работы [14], на которую мы ссылаемся, относятся к $\mathbb{R}^{n}$, но цитированный результат (и многое другое) вместе с его доказательством, конечно, сохраняется на любом римановом многообразии. Вот это доказательство:

Пусть $\bmod (\Gamma)=0$. Тогда существует такая последовательность $\left\{\rho_{k}\right\}$ допустимых для $\Gamma$ функций, что $\int_{M} \rho_{k}^{n}<4^{-k}$. Положим $\rho=\left(\sum_{k=1}^{\infty} 2^{k} \rho_{k}^{n}\right)^{1 / n}$. Тогда $\int_{M} \rho^{n}<\infty$ и в то же время $\int_{\gamma} \rho \geqslant \int_{\gamma} 2^{k / n} \rho_{k} \geqslant 2^{k / n}$ для любого натурального $k$ и любой кривой $\gamma \in \Gamma$.

Отметим еще, что построенная функция $\rho$, вообще говоря, может где-то обращаться в нуль. Чтобы устранить вырождение метрики $\rho^{2} g$, можно добавить к $\rho^{n}$ любую положительную функцию $p \in\left(C^{\infty} \cap L^{n}\right)(M)$ и заменить $\rho$ на $\rho_{+}=\left(\rho^{n}+p\right)^{1 / n}$. Функцию $p$, очевидно, можно выбрать той же степени гладкости, что и разбиение единицы на $(M, g)$, т. е. степени гладкости самого многообразия.

Наконец, естественно сгладить (например, осреднением) саму функцию $\rho_{+}$. Чтобы избежать возникающих при этом технических осложнений, связанных с рассмотрением интегралов на кривых $\gamma$ семейства $\Gamma$, здесь удобнее перейти на язык емкостей и, вернувшись к началу, повторить все проведенное рассуждение в терминах исчерпывающей $M$ последовательности колец $R_{k}=R_{r_{k}}^{r_{k+1}} \subset M$, таких, что $\sum$ cap $R_{k}<\infty$. В разд. 2.3 было отмечено, что почти экстремальные функции $U_{k}$ для емкостей колец $R_{k}$ можно брать столь же гладкими, как и само многообразие. Это же относится и к $U=\sum U_{k}$. Теперь можно положить $\lambda=\|\operatorname{grad} U\|$ и перейти к искомой гладкой метрике $\lambda^{2} g$ конечного объема, устранив, как было показано выше, обращение $\lambda$ в нуль, если такое вырождение имеется.

\section{§4. Некоторые общие интегральные соотношения на римановом многообразии}

4.1. Изопериметрическая функция. Функция $P: \mathbb{R}_{+} \rightarrow \mathbb{R}_{+}$называется изопериметрической функиией $n$-мерного риманова многообразия $(M, g)$, если для любой области $D \subset M$ с регулярной границей $\partial D$ выполнено соотношение

$$
P\left(\operatorname{vol}_{n} D\right) \leqslant \operatorname{vol}_{n-1}(\partial D) .
$$

Например, функции $P(v)=v^{(n-1) / n}$ и $P(v)=v$ с точностью до постоянного множителя являются изопериметрическими для $\mathbb{R}^{n}$ и $\mathbb{H}^{n}$ соответственно. 
Изопериметрических (не максимальных) функций на многообразии, вообще говоря, много. Тривиальный пример: $P \equiv 0$.

Если неравенство (6) выполнено только для специального семейства областей, то говорят, что $P$ - изопериметрическая функция этого семейства.

В качестве такого семейства ниже рассматривается совокупность геодезических (в метрике $g$ ) шаров $B\left(x_{0}, r\right)$ радиуса $r$ с фиксированным центром $x_{0}$. Это семейство мы будем называть геодезическим, шаровым или сферическим исчерпанием многообразия $(M, g)$, поскольку нас будут интересовать большие шары $B\left(x_{0}, r\right)$, сферы $\partial B\left(x_{0}, r\right)$ и кольца $R_{r_{0}}^{r}=B\left(x_{0}, r\right) \backslash \bar{B}\left(x_{0}, r_{0}\right)$.

Величины $\operatorname{vol}_{n} B\left(x_{0}, r\right), \operatorname{vol}_{n-1} \partial B\left(x_{0}, r\right)$ для краткости будут обозначаться через $v(r)$ и $S(r)$ соответственно. Тогда

$$
v(r)=\int_{0}^{r} S(t) d t
$$

При переходе от метрики $g$ к метрике $\tilde{g}$ соответствующие объекты будут наделяться «тильдой».

4.2. Изопериметрическая функция и конформная емкость. На $n$-мерном римановом многообразии $(M, g)$ справедливо (см., например, [32, разд. 2.2.3]) следующее соотношение:

$$
\left(\int_{v\left(r_{0}\right)}^{v\left(r_{1}\right)} P^{n /(1-n)}\right)^{1-n} \leqslant \operatorname{cap} R_{r_{0}}^{r_{1}} .
$$

СлЕДСТВИЕ 1. Для конформной параболичности (гиперболичности) $n$-мерного риманова многообразия $(M, g)$ необходимо (достаточно), чтобь расходился (сходился) интеграл

$$
\int^{\bullet} P^{n /(1-n)}
$$

Здесь имеется в виду сходимость интеграла на верхнем пределе, т. е. в окрестности правого (верхнего) конца области определения функции $P$.

ЗАмЕЧАниЕ 1. Если в (8) универсальную изопериметрическую функцию заменить изопериметрической функцией геодезических шаров сферического исчерпания, то неравенство (8), вообще говоря, может нарушиться. Это будет попутно показано ниже в $\S 6$, замечание 7 .

4.3. Площади геодезических сфер и емкость колец. На $n$-мерном римановом многообразии $(M, g)$ справедливо соотношение

$$
\operatorname{cap} R_{r_{0}}^{r_{1}} \leqslant\left(\int_{r_{0}}^{r_{1}} S^{1 /(1-n)}\right)^{1-n} .
$$

СлЕДСТВИЕ 2. Для конформной параболичности (гиперболичности) $n$-мерного риманова многообразия $(M, g)$ достаточно (необходимо), чтобь расходился (сходился) интеграл

$$
\int^{\bullet} S^{1 /(1-n)}
$$


Здесь имеется в виду сходимость интеграла в окрестности правого конца промежутка, где определена функция $S(r)$ (т. е. в области относительно больших значений $r$ ).

Если многообразие геодезически полно, то речь идет о сходимости или расходимости этого интеграла в окрестности бесконечности.

Доказательство неравенства (9) получается непосредственным предъявлением функции

$$
U(r)=\left(\int_{r_{0}}^{r} S^{1 /(1-n)}\right)\left(\int_{r_{0}}^{r_{1}} S^{1 /(1-n)}\right)^{-1},
$$

допустимой для кольца $R_{r_{0}}^{r_{1}}\left(U\left(r_{0}\right)=0\right.$ и $\left.U\left(r_{1}\right)=1\right)$.

ЗАмЕчАНИЕ 2. Полезно заметить, что если метрика $g$ сферически симметрична (относительно центра $x_{0}$ ), т. е. $g$ зависит только от расстояния $r$ до $x_{0}$ $\left(\right.$ как в $\mathbb{R}^{n}$ и $\mathbb{H}^{n}$ ), то предъявленная функция $U(r)$ экстремальна и в $(9)$ имеет место равенство.

В сферически симметричных пространствах сформулированные в следствии 2 признаки становятся критериями конформного типа многообразия.

Объединяя (8) и $(9)$, получаем соотношение

$$
\left(\int_{v\left(r_{0}\right)}^{v\left(r_{1}\right)} P^{n /(1-n)}\right)^{1-n} \leqslant \operatorname{cap} R_{r_{0}}^{r_{1}} \leqslant\left(\int_{r_{0}}^{r_{1}} S^{1 /(1-n)}\right)^{1-n} .
$$

Отметим, что для $n=2$ утверждения, высказанные в следствиях 1 и 2 , впервые были доказаны Альфорсом в $[1,2]$.

\section{4. Конформная замена метрики и лемма Альфорса-Громова.} При переходе от метрики $g$ к конформной ей метрике $\tilde{g}=\lambda^{2} g$ емкость кольца $R_{r_{0}}^{r_{1}}$ как конденсатора $R\left(C_{0}, C_{1}\right)$, конечно, сохраняется, но оно, вообще говоря, перестает быть областью вида $R_{\tilde{r}_{0}}^{\tilde{r}_{1}}$, ограниченной сферами радиусов $\tilde{r}_{0}$ и $\tilde{r}_{1}$ в новой метрике $\tilde{g}$. Переходы, сохраняющие систему сфер с данным центром, очевидно, имеют вид

$$
\tilde{g}=\lambda^{2}(r) g \text {. }
$$

Для таких специальных, сферически конформных метрик

$$
\begin{aligned}
\tilde{r} & =\int_{0}^{r} \lambda(t) d t, \\
\tilde{S}(\tilde{r}) & =\lambda^{n-1}(r) S(r), \\
\tilde{v}(\tilde{r}) & =\int_{0}^{r} \lambda^{n}(t) S(t) d t, \\
\widetilde{S}^{1 /(1-n)}(\tilde{r}) d \tilde{r} & =S^{1 /(1-n)}(r) d r, \\
\int_{\tilde{r}_{0}}^{\tilde{r}_{1}} \widetilde{S}^{1 /(1-n)}(\tilde{r}) d \tilde{r} & =\int_{r_{0}}^{r_{1}} S^{1 /(1-n)}(r) d r .
\end{aligned}
$$

Значит, для метрик $\tilde{g}$ вида $\lambda^{2}(r) g$ инвариантны обе части неравенства $(9)$.

Сопоставление соотношений $(10)$, записанных для $\tilde{g}$ и $g$, в случае конформной метрики общего вида $\tilde{g}=\lambda^{2} g$ ввиду неинвариантности сфер невозможно. 
Тем не менее имеет место следующая восходящая к Альфорсу [2, с. 186] и в почти таком же, как приводимый ниже, виде рассмотренная Громовым $[19$, с. $198 ; 20$, с. 85] (см. также [36])

ЛЕмма 1. Если римановы многообразия $(M, g),(M, \tilde{g})$ конформны, то

$$
\int_{\tilde{v}\left(r_{0}\right)}^{\tilde{v}\left(r_{1}\right)} \widetilde{P}^{n /(1-n)} \geqslant \int_{r_{0}}^{r_{1}} S^{1 /(1-n)}
$$

где $\widetilde{P}$ - любал изопериметрическал функиия метрики $\tilde{g}, a \tilde{v}\left(r_{0}\right), \tilde{v}\left(r_{1}\right)$ суть $\tilde{g}$-объемь геодезических g-шаров радиусов $r_{0}$ u $r_{1}$ соответственно.

Дадим короткое доказательство этой леммы и сделаем по его ходу одно используемое ниже замечание. Так как $\tilde{g}=\lambda^{2} g$, то, обозначив через $\tilde{v}(t)$ и $\widetilde{S}(t)$ $\tilde{g}$-меру $g$-шара радиуса $t$ и $g$-сферы радиуса $t$ соответственно, имеем

$$
\tilde{v}(t)=\int_{0}^{t} d \tau \int_{\partial B(\tau)} \lambda^{n} d \sigma, \quad \widetilde{S}(t)=\int_{\partial B(t)} \lambda^{n-1} d \sigma,
$$

где $d \sigma$ - элемент $(n-1)$-меры в метрике $g$. По неравенству Гёльдера

$$
\widetilde{S}^{n}(t)=\left(\int_{\partial B(t)} \lambda^{n-1} d \sigma\right)^{n} \leqslant\left(\int_{\partial B(\tau)} \lambda^{n} d \sigma\right)^{n-1}\left(\int_{\partial B(t)} d \sigma\right)=\left(\tilde{v}^{\prime}(t)\right)^{n-1} S(t),
$$

а в силу изопериметрического неравенства $\widetilde{P}(\tilde{v}) \leqslant \widetilde{S}$ (используемого здесь только для $g$-шаров и сфер) получаем

$$
\widetilde{P}^{n}(\tilde{v}(t)) \leqslant\left(\tilde{v}^{\prime}(t)\right)^{n-1} S(t)
$$

или

$$
S^{1 /(1-n)}(t) \leqslant \widetilde{P}^{n /(1-n)}(\tilde{v}(t)) \tilde{v}^{\prime}(t) .
$$

Интегрируя последнее неравенство в пределах от $r_{0}$ до $r_{1}$, получаем (17).

ЗАмЕчАНИЕ 3. Из приведенного доказательства видно, что лемма остается в силе, даже если $\widetilde{P}$ - не универсальная изопериметрическая функция в метрике $\tilde{g}$, а является таковой только для $g$-шаров рассматриваемого семейства.

Полезно сопоставить это замечание с замечанием 1 и соотношением (10), чтобы оценить роль (17) наряду с (10).

\section{§5. Мера геодезических сфер и конформный тип многообразия}

Рассмотрим подробнее правую часть соотношения (10), т. е. неравенство (9).

5.1. Критерий Альфорса. Обобщая результаты Кобаяси и Неванлинны, Альфорс в [1] впервые показал, что расходимость интеграла $\left(9^{\prime}\right)$ при $n=2$ является достаточным признаком параболичности односвязной открытой поверхности в классическом смысле теории униформизации, т.е. в смысле возможности ее конформного отображения на всю комплексную плоскость.

Более того, Альфорс заметил, что в конформном классе метрики поверхности параболического типа всегда найдется такая метрика, в которой интеграл $\left(9^{\prime}\right)$ расходится. 
Это красивое наблюдение может служить некоторым основанием для трансформации получаемого таким образом критерия параболичности в определение конформной параболичности любого $n$-мерного риманова многообразия, как это сделано в [21], где затем доказана эквивалентность такого определения приведенному во введении определению в терминах конформной емкости.

Итак, справедлив следующий критерий Альфорса конформной параболичности риманова многообразия: риманово многообразие $(M, g)$ конформно-параболично тогда и только тогда, когда существует такая метрика $\tilde{g}$, конбормная метрике $g$, в которой многообразие $(M, \tilde{g})$ полно и

$$
\int^{\infty} \widetilde{S}^{1 /(1-n)}=\infty
$$

Приведем доказательство этого критерия, опирающееся на изложенные здесь факты.

Неравенство (9), как уже отмечалось, говорит о достаточности условия $\left(9^{\prime \prime}\right)$. А доказанный в $\S 3$ критерий параболичности показывает, что если взять такую полную метрику, в которой объем многообразия $M$ конечен, то в силу равенства $\operatorname{vol}(M)=\int_{0}^{\infty} S(r) d r<\infty$ очевидно, что

$$
\int^{\infty} S^{-\alpha}(r) d r=\infty
$$

при любом $\alpha>0$, в том числе и при $\alpha=1 /(n-1)$.

5.2. Некоторые достаточные признаки параболичности. О расходимости интеграла $\left(9^{\prime}\right)$ можно судить по некоторым иным иногда удобным признакам, на которых мы здесь остановимся.

Отметим сначала следующее общее неравенство:

$$
\int_{r 0}^{r_{1}}\left(\frac{r-r_{0}}{v(r)}\right)^{1 /(n-1)} d r \leqslant n^{1 /(n-1)} \int_{r_{0}}^{r_{1}}\left(\frac{1}{S(r)}\right)^{1 /(n-1)} d r,
$$

где $r_{0}<r_{1}, 0<v(r), 0<S(r)$ и $v^{\prime}(r)=S(r)$.

Для его доказательства достаточно по неравенству Гёльдера написать

$$
\begin{aligned}
& \int_{r_{0}}^{r}\left(\frac{r-r_{0}}{v(r)}\right)^{1 /(n-1)} d r \\
& \quad \leqslant\left(\int_{r_{0}}^{r}\left(\frac{r-r_{0}}{v(r)}\right)^{n /(n-1)} v^{\prime}(r) d r\right)^{1 / n}\left(\int_{r_{0}}^{r}\left(\frac{1}{S(r)}\right)^{1 /(n-1)} d r\right)^{(n-1) / n},
\end{aligned}
$$

проинтегрировать по частям первый множитель справа

$$
\begin{aligned}
\int_{r_{0}}^{r_{1}}\left(\frac{r-r_{0}}{v(r)}\right)^{n /(n-1)} v^{\prime}(r) d r & \\
& =\left.(1-n)\left(\frac{\left(r-r_{0}\right)^{n}}{v(r)}\right)^{1 /(n-1)}\right|_{r_{0}} ^{r_{1}}+n \int_{r_{0}}^{r_{1}}\left(\frac{r-r_{0}}{v(r)}\right)^{1 /(n-1)} d r,
\end{aligned}
$$

учесть отрицательность первого слагаемого справа

$$
\int_{r_{0}}^{r_{1}}\left(\frac{r-r_{0}}{v(r)}\right)^{n /(n-1)} v^{\prime}(r) d r \leqslant n \int_{r_{0}}^{r_{1}}\left(\frac{r-r_{0}}{v(r)}\right)^{1 /(n-1)} d r
$$


и, продолжив первое неравенство с учетом последнего, после сокращений получить (18).

Соотношения (9) и (18) позволяют высказать следующее

ПРЕДЛОЖЕНИЕ 1 . Для того чтобы $n$-мерное риманово многообразие $(M, g)$ было конформно-параболического типа, достаточно, чтобы интеграл

$$
\int^{\omega}\left(\frac{r}{v(r)}\right)^{1 /(n-1)} d r
$$

аде $v(r)$ - объем геодезического шара g-радиуса $r$, расходился на верхнем пределе $\omega$ области определения.

(Если метрика $g$ полная, то $\omega=\infty$.)

СлЕДСТВИЕ 3. Если п-мерное полное риманово многообразие $(M, g)$ таково, что

$$
\liminf _{r \rightarrow+\infty} \frac{v(r)}{r^{n}}<\infty
$$

то оно конформно-параболично.

Для доказательства, учитывая (20), найдем $c>0$ и последовательность $r_{k}$, такие, что $r_{k} \rightarrow+\infty$ и $v\left(r_{k}\right)<c r_{k}^{n}$. Переходя, если нужно, к подпоследовательности, можно считать, что $2 r_{k}<r_{k+1}$. Тогда

$$
\begin{aligned}
\int_{r_{1}}^{+\infty}\left(\frac{r}{v(r)}\right)^{1 /(n-1)} d r & >\sum_{k=1}^{\infty} \int_{r_{k}}^{r_{k+1}}\left(\frac{r}{c r_{k+1}^{n}}\right)^{1 /(n-1)} d r \\
& =\frac{n-1}{n c^{1 /(n-1)}} \sum_{k=1}^{\infty} r_{k+1}^{n /(1-n)}\left(r_{k+1}^{n /(n-1)}-r_{k}^{n /(n-1)}\right) \\
& =\frac{n-1}{n c^{1 /(n-1)}} \sum_{k=1}^{\infty}\left(1-\left(\frac{r_{k}}{r_{k+1}}\right)^{n /(n-1)}\right) .
\end{aligned}
$$

Последний ряд, а с ним и интеграл (19) расходятся.

ЗАмЕчАНИЕ 4. Для сферически симметричных римановых многообразий (т. е. с метрикой, зависящей только от расстояния $r$ до фиксированной точки), на которых функция $S(r)$, например, не убывает, расходимость интеграла (19) является не только достаточным, но и необходимым условием параболичности.

Действительно, учитывая, что $v^{\prime}(r)=S(r)$, в этом случае имеем

$$
v(r)=v(r)-v(0) \leqslant S(r) r,
$$

откуда

$$
\int_{r_{0}}^{r_{1}}\left(\frac{r}{v(r)}\right)^{1 /(n-1)} d r \geqslant \int_{r_{0}}^{r_{1}}\left(\frac{1}{S(r)}\right)^{1 /(n-1)} d r .
$$

Остается воспользоваться замечанием 2.

5.3. Роль конформного класса метрики. Полученные признаки конформной параболичности риманова многообразия в конформном классе его метрики можно, используя в качестве инструмента наблюдение (см. $\S \S 1$ и 3) о 
полной метрике конечного объема, превратить в критерии, подобно тому как это было сделано выше при доказательстве критерия Альфорса.

Условимся говорить, что некоторое свойство или соотношение реализуемо $в$ каком-то классе метрик, если оно реализуется, т. е. выполнено для некоторой метрики этого класса.

Например, любое риманово многообразие $(M, g)$ по отношению к конформному классу его метрики реализуемо как полное, т.е. класс полных метрик, конформных метрике $g$, непуст (это видно из равенства (12)).

Tеорема 1. Риманово многообразие $(M, g)$ конформно-параболично тогда и только тогда, когда в классе полных метрик, конформньх метрике $g$, реализуемь следующие эквивалентнье между собой условия

(i) $\operatorname{vol}(M)<\infty$,

(ii) $\int^{\infty} S^{1 /(1-n)}(r) d r=\infty$,

(iii) $\int^{\infty}\left(\frac{r}{v(r)}\right)^{1 /(n-1)} d r=\infty$,

(iv) $\liminf _{r \rightarrow \infty} \frac{v(r)}{r^{n}}<\infty$.

Критерии (i), (ii) уже доказаны выше в 3 и разд. 5.1 соответственно. Достаточность (iii) и (iv) только что обсуждалась в разд. 5.2. Их необходимость, как и в случае (ii), видна из (i).

Отметим, что в индивидуальной метрике, как было показано выше, справедливы импликации (iv) $\Longrightarrow$ (iii) $\Longrightarrow$ (ii) и, очевидно, (i) $\Longrightarrow$ (iv), хотя выполнение (i), конечно, влечет за собой и более сильные соотношения вида (ii), (iii), (iv) (например, (iv) в этом случае выполнено при любом $n>0$ ).

В индивидуальной метрике условия (i)-(iv) не эквивалентны.

ЗАмЕчАНИЕ 5. Завершая анализ правой части соотношения (10), подчеркнем, что указанные в теореме критерии параболичности не являются критериями в индивидуальной метрике. Например, сходимость интеграла из условия (ii) теоремы 1 в индивидуальной метрике вовсе не означает гиперболичности многообразия. Более того, как показано в [21], в конформном классе любого, в том числе параболического, многообразия всегда есть такая полная метрика, в которой этот интеграл сходится. В работе [21] дано полное описание функций $S(r)=v^{\prime}(r)$, точнее, дано полное описание функций $v(r)$, которые могут появиться в конформном классе метрик многообразий параболического и гиперболического типов.

\section{§6. Изопериметрическая функция и конформный тип многообразия}

\section{1. Изопериметрическая функция сферического исчерпания в} конформном классе метрики. Вернемся теперь к левой части соотношения (10) и обсудим некоторые свойства изопериметрической функции в конформном классе метрик различных конформных типов.

Сохраняя обозначения и терминологию, введенные в разд. 4.1, рассмотрим сферическое исчерпание многообразия $(M, g)$, т. е. совокупность геодезических 
шаров $B\left(x_{0}, r\right) g$-радиуса $r$ с обшим центром $x_{0}$. Эта система шаров инвариантна относительно перехода к метрике $\tilde{g}$ вида $\lambda^{2}(r) g$, сферически конформной метрике $g$. Меры $\operatorname{vol}_{n} B\left(x_{0}, r\right)$ и $\operatorname{vol}_{n-1} \partial B\left(x_{0}, r\right)$ в метриках $g$ и $\tilde{g}$ обозначим соответственно через $v(r), S(r)$ и $\tilde{v}(r), \widetilde{S}(r)$.

Пусть $I$ - открытый (конечный или бесконечный) промежуток на полуоси $\mathbb{R}_{+}$положительных чисел, на котором задана неотрицательная функция $P \in C^{\infty}(I, \mathbb{R})$. (Из дальнейшего будет видно, что $P$ можно даже считать имеющей порядок гладкости многообразия $M$. Этого достаточно для того, чтобы проводимая ниже конформная замена метрики не понижала гладкость многообразия $M$.)

ПРЕДЛОЖЕНИЕ 2. Функиия $P$ является изопериметрической функиией, причем максимальной (т.е. вида $P(\tilde{v})=\widetilde{S})$ для всех достаточно больших шаров сферического исчерпания риманова многообразия в некоторой метрике, сферически конформной исходной, тогда и только тогда, когда интегральь

$$
\text { (a) } \int^{\bullet} P^{n /(1-n)}, \quad \text { (b) } \int^{\bullet} S^{1 /(1-n)}
$$

сходятся или расходятся одновременно на верхнем пределе области определения своей подьнтегральной функиии.

При этом новая метрика будет полной тогда и только тогда, когда $\int^{\bullet} P^{-1}=\infty$.

Для доказательства заметим сначала, что если $P(v)=S$, то, учитывая, что $v^{\prime}(r)=S(r)$, получаем равенство

$$
\int_{v\left(r_{0}\right)}^{v\left(r_{1}\right)} P^{n /(1-n)}=\int_{r_{0}}^{r_{1}} S^{1 /(1-n)} .
$$

Если соотношение $P(\tilde{v})=\widetilde{S}$ имеет место в некоторой метрике $\tilde{g}$ вида $\lambda^{2}(r) g$, то, переписав (22) для $\tilde{g}$ и учитывая (16) и принятые здесь обозначения, получаем

$$
\int_{\tilde{v}\left(r_{0}\right)}^{\tilde{v}\left(r_{1}\right)} P^{n /(1-n)}=\int_{r_{0}}^{r_{1}} S^{1 /(1-n)} .
$$

Если интегралы (21) расходятся, то, фиксировав произвольно $\tilde{v}\left(r_{0}\right) \in I$ и меняя $r_{1}$, из равенства (23) однозначно найдем функцию $\tilde{v}(r)$ для $r>r_{0}$.

Если же интегралы (21) сходятся, то фиксируем $\tilde{v}\left(r_{1}\right)$ и $r_{1}$ на верхних концах областей определения соответствующих подынтегральных функций и, меняя на сей раз $r_{0}$, опять найдем $\tilde{v}(r)$ для всех допустимо больших значений $r$.

Зная $\tilde{v}(r)$ и вспомнив формулы (12)-(14), с учетом обозначения $\tilde{v}(r)=\tilde{v}(\tilde{r}(r))$, получаем

$$
\lambda^{n}(r)=\left(\tilde{v}^{\prime} / S\right)(r)
$$

для всех допустимо больших значений $r$.

В область меньших значений $r$ функцию $\lambda(r)$ можно продолжить так, чтобы для некоторого $r_{0}>0$ и соответствующего значения $\tilde{v}\left(r_{0}\right) \in I=(\alpha, \beta)$ значения интегралов $(21 \mathrm{~b})$ и $(21 \mathrm{a})$, взятых по промежуткам $\left(0, r_{0}\right)$ и $\left(\alpha, \tilde{v}\left(r_{0}\right)\right)$ соответственно, совпали. 
Таким образом, при условии, что интегралы (21) сходятся или расходятся одновременно, найдена метрика $\tilde{g}=\lambda^{2}(r) g$, в которой заданная функция $P$ является изопериметрической вида $P(\tilde{v})=\widetilde{S}$ для всех достаточно больших шаров рассматриваемого сферического исчерпания риманова многообразия.

Тот факт, что сформулированные условия на интегралы (21) необходимы, очевиден в силу равенства (22).

Наконец, о полноте новой метрики $\tilde{g}$. Поскольку $d \tilde{v}=\widetilde{S}(\tilde{r}) d \tilde{r}=P(\tilde{v}) \lambda(r) d r$ (см. (12)-(14)), то интегралы $\int^{\bullet} \lambda, \int^{\bullet} P^{-1}$ действительно сходятся или расходятся одновременно.

ЗАмЕчАНИЕ 6. Если не фиксировать вид $P(v)=S$ изопериметрического соотношения, а требовать только неравенства $P(v) \leqslant S$, то в силу леммы Альфорса-Громова (17) и замечания 3 к ней сходимость интеграла (21a) влечет за собой сходимость интеграла (21b). Однако одновременной сходимости или расходимости этих интегралов при соотношении $P(v) \leqslant S$, конечно, может и не быть: например, в случае $P(v) \equiv 0$.

Таким образом, условие $P(v)=S$ максимальности изопериметрической функции в предложении 2 существенно.

ЗАмЕЧАНИЕ 7. Предложение 2 можно использовать, чтобы реализовать теперь сказанное в замечании 1 . Покажем, что если $P$ - не универсальная изопериметрическая функция, а всего лишь изопериметрическая функция для шаров сферического исчерпания, то неравенство (8), т. е. левая часть соотношений (10), может нарушиться.

Действительно, например, на многообразии параболического типа, как уже отмечалось со ссылкой на [21], можно взять полную метрику, для которой интеграл (21b) сходится в бесконечности. Эта сходимость ввиду (16) сохранится при переходе к любой сферически конформной ей метрике; поэтому на основании предложения 2 можно считать, что для всех достаточно больших шаров нашего исчерпания выполняется соотношение $P(v)=v=S$. Равенство (22) показывает, что в рассматриваемой ситуации значения крайних членов соотношения (10) совпадают. Однако по условию они не стремятся к нулю при $r_{1} \rightarrow+\infty$ в отличие от среднего члена.

\section{2. Изопериметрические признаки конформного типа многообра- зия.}

ПРЕДЛОЖЕНИЕ 3. (i) Для риманова многообразия $(M, g)$ конформно-параболического типа любая неотричательная функиия $P \in C^{\infty}(I, \mathbb{R})$ является максимальной изопериметрической функчией $\tilde{g}$-сферического исчерпания многообразия $M$ в некоторой метрике $\tilde{g}$, конформной метрике $g$.

Если интеграль (21a), (21b) сходятся или расходятся одновременно, то $\tilde{g}$ можно взять вида $\lambda^{2}(r) g$.

(ii) Для риманова многообразия $(M, g)$ конформно-гиперболического типа неотричательная функчия $P \in C^{\infty}(I, \mathbb{R})$ является максимальной изопериметрической функиией сферического исчерпания многообразия $M$ в некоторой метрике $\tilde{g}=\lambda^{2}(r) g$, сферически конформной метрике $g$, тогда $u$ только тогда, когда интеграл (21a) сходитсл.

Сходимость интеграла (21b) является по следствию 2 необходимым условием гиперболичности многообразия. Значит, достаточность в п. (ii) предложения 3 
непосредственно вытекает из предложения 2. Необходимость же следует из равенства (23).

Чтобы доказать п. (i), достаточно вспомнить, что на многообразии конформно-параболического типа есть такие метрики, для которых интеграл (21b) расходится (см. выше критерий Альфорса или [21]), и есть конформно инвариантные им метрики, для которых он сходится (см. [21]). Остается применить предложение 2.

Некоторой детализацией пп. (i) и (ii) предложения 3 являются следующие два предложения 4 и 5 соответственно.

ПРЕДЛОЖЕНИЕ 4. (i) Если $n$-мерное риманово многообразие $(M, g)$ конформно-параболично, то найдется полная метрика $\tilde{g}$, конформная метрике $g$, в которой максимальная изопериметрическая функиия для больших шаров $\tilde{g}$-сферического исчерпания $M$ имеет вид $P(\tilde{v})=\tilde{v}^{(n-1) / n}\left(\kappa a \kappa в ~ \mathbb{R}^{n}\right)$.

(ii) Если максимальная изопериметрическая функиия сферического исчерпания п-мерного риманова многообразия $(M, g)$ в полной метрике $g$ для больших шаров имеет вид $P(v)=v^{(n-1) / n}$, то многообразие $(M, g)$ конформно-параболично.

Для доказательства п. (i) сначала фиксируем на $(M, g)$ такую полную метрику, конформную метрике $g$, для которой интеграл (21b) расходится в бесконечности, как и интеграл (21а) для рассматриваемой функции $P(v)=v^{n /(n-1)}$. Например, можно считать, что сама исходная метрика $g$ есть полная метрика конечного объема: для нее интеграл (21b), очевидно, расходится в бесконечности (см. разд. 5.1). В силу предложения 3 найдется метрика $\tilde{g}$ вида $\lambda^{2}(r) g$, в которой данная функция $P$ будет изопериметрической функцией $\tilde{g}$ - $($ и $g$-)сферического исчерпания, заданной на бесконечном промежутке $I=(0,+\infty)$. Метрика $\tilde{g}$ при этом будет полной в силу заключительной части предложения 2.

Для доказательства п. (ii) заметим сначала, что величина $S(r)$ отделена от нуля при $r \rightarrow+\infty$, так как $v^{(n-1) / n}=S$. Значит, $\operatorname{vol}_{g}(M)=\int_{0}^{\infty} S=\infty$, и интеграл (21a) для данной функции $P$ расходится в бесконечности. Ввиду равенства (22) интеграл (21b) тоже должен расходиться в бесконечности. Тогда по следствию $2(M, g)$ конформно-параболично.

ЗАМЕЧАНИЕ 8 . В п. (ii) предложения 4 равенство $v^{(n-1) / n}=S$, выполненное для больших сфер, как и вообще равенство $P(v)=S$, конечно, можно заменить на $P(v)=c S$, где $c$ - переменная, но отделенная от 0 и $\infty$ величина.

ПРЕДЛОЖЕНИЕ 5. (i) Если риманово многообразие $(M, g)$ конформно-гиперболично, то найдется сферически конформная метрике $g$ метрика $\tilde{g}=$ $\lambda^{2}(r) g$, в которой максимальная изопериметрическая функиия сферического исчерпания имеет вид $P(v)=v\left(\kappa a \kappa в \mathbb{H}^{n}\right)$.

(ii) Если риманово многообразие $(M, g)$ таково, что для яюбой метрики, конформной метрике $g$, найдетсл полная сферически ей конформная метрика, в которой максимальная изопериметрическая функиия имеет вид $P(v)=v$, то многообразие $(M, g)$ конформно-гиперболично.

Пункт (i) - конкретизация п. (ii) предложения 3.

Для доказательства п. (ii) заметим, что по лемме 1 интеграл (21b) должен сходиться в любой метрике, конформной данной. В силу критерия Альфорса (см. разд. 5.1) отсюда следует гиперболичность данного многообразия. 


\section{§7. Заключительные замечания}

\section{1. Параболичность многообразия в теории дифференциальных} уравнений и конформная параболичность. В теории гармонических функций и теории оператора Лапласа-Бельтрами понятие параболичности относится к римановым многообразиям, на которых имеет место теорема лиувиллиева типа о постоянстве (суб)гармонической функции. В размерности $n=2$, где интеграл Дирихле конформно-инвариантен, такое понимание параболичности совпадает с конформной параболичностью, обсуждавшейся выше. В старших размерностях это не так. В теории так называемых $p$-(суб)гармонических функций вводят адекватное понятие $p$-емкости. Классическое уравнение Лапласа при этом отвечает случаю $p=2$, а конформно-инвариантная теория - случаю $p=n$. Таким образом, $(p=n)$-параболичность в теории нелинейного аналога уравнения Лапласа соответствует рассмотренной здесь конформной параболичности риманова многообразия. О параболичности с точки зрения комплексного анализа и теории дифференциальных уравнений см., например, $[1,2,5,35,40]$ и $[8,18,24-28,33,42]$ соответственно.

7.2. Другие геометрические признаки параболичности. Наряду с описанными здесь или в уже цитированной выше литературе имеются и другие геометрические признаки параболичности. Например, Милнор описал связь между конформным типом поверхности, ее кривизной, порядком роста фундаментальной группы базы и скоростью роста объема геодезических шаров накрытия $[30,31]$. В этой связи см. также [41] и гл. 6 в [20].

7.3. Конформные инварианты и реализация идеальной границы. Конформный тип риманова многообразия по существу определяется его строением на бесконечности, точнее, емкостью его идеальной границы - абсолюта. На конформных моделях пространств $\mathbb{R}^{n}$ и $\mathbb{H}^{n}$ в виде проколотой сферы $\dot{S}^{n}$ и единичного шара $B^{n}$ соответственно абсолют хорошо реализуется (точка и сфера соответственно) и вопрос о его емкости (нуль или не нуль) становится тривиальным. В принципе идеальную границу можно конформно-инвариантно реализовать и в случае общего риманова многообразия (например, как это отмечалось в $[47$, с. 141]). Используя конформные инварианты (емкость, модуль или экстремальную длину), на многообразии вводят конформно-инвариантную метрику (см., например, $[6,22,11-13])$ и пополняют многообразие по этой метрике. В размерности $n=2$ это пополнение совпадает с классической компактификацией в смысле Каратеодори, которая служит для описания соответствия границ при конформных отображениях. Идея такого использования указанных конформных инвариантов и сами они связаны с именем Альфорса $([4,3,39])$, как и многое в изложенном выше. Сравнительно недавнее красивое применение этих конформных инвариантов, связанное с магнитной гидродинамикой и узлами, описано в $[9,10]$.

\section{ЛитературА}

1. Ahlfors L. Sur le type d'une surface de Riemann. C. R. Acad. Sci. Paris Ser. A, 201, 30-32 (1935).

2. Ahlfors L. Zur Theorie des Überlagerungsflächen, Acta Math., 65, 157-194 (1935). 
3. Ahlfors L. Conformal invariants (Topics in Geometric Function Theory). McGrowHill, New York, 1973.

4. Ahlfors L., Beurling A. Conformal invariants and function-theoretic null-sets. Acta Math., 83, 101-129 (1950).

5. Ahlfors L., Sario L. Riemann surfaces. Princ. Univ. Press, Princeton, 1960.

6. Anderson G. D., Vamanamurthy M. K., Vuorinen M. Conformal invariants, quasiconformal maps and special functions. Lecture Notes in Math., Vol. 1508, 1992, p. $1-19$.

7. Caraman P. $n$-Dimensional Quasiconformal Mappings. Editura Academiei Romane, Bucharest, Abacus Press, Tunbridge Wells Haessner Publishing, Inc., Newfoundland, N. J., 1974.

8. Cheng S. V., Yau S. T. Differential equations on Riemannian manifolds and their geometric applications. Comm. Pure Appl. Math., 28, 333-354 (1975).

9. Freedman M. H., He Z. H. Divergence free fields: Energy and asymptotic crossing number. Ann. Math., 134, 189-229 (1991).

10. Freedman M. H., He Z. H. Links of tori and the energy of incompressible flows. Topology, 30, No. 2, 283-287 (1991).

11. Lelong-Ferrand $J$. Invariant conform globaux sur les variétés Riemanniennes. J. Diff. Geom., 8, 487-510 (1973).

12. Ferrand $J$. Conformal capacity and conformally invariant metrics. To appear.

13. Ferrand $J$. Conformal capacity and conformally invariant functions on manifolds. C. R. Acad. Sci. Paris Sér. I, 218, 213-216 (1994).

14. Fuglede B. Extremal lenght and functional completion. Acta Math., 83, 101-129 (1957).

15. Gehring F. V. Symmetrization of rings in space. Trans. Am. Math. Soc., 101, 499-519 (1961).

16. Gehring F. V. Extremal lenght definitions for the conformal capacity of rings in space. Mich. Math. J., 9 (162), 137-150.

17. Gehring F. V. Topics in quasiconformal mappings. Lecture Notes in Math. Vol. 1508, 20-38 (1992); Reprinted from Proc. Internat. Congr. Math. (Berkeley, California 1986), Vol. 1, Am. Math. Soc., 1987, pp. 62-80.

18. Григорьян A. A. О существовании положительных фундаментальных решений уравнения Лапласа на римановых многообразиях. Матем. сб., 128 (170), №3 (11), 354-363 (1985).

19. Gromov M. Hyperbolic manifolds, groups and actions. Proceedings of the 1978 Stony Brook Conference, Ann. of Math. Studies, Vol. 97, Princeton Univ. Press, Princeton, N. J., 1981, pp. 183-213.

20. Gromov M. Structures métriques pour les variétés riemanniennes. Notes du cours rédigé par J. Lafontaine et P. Pansu, CEDIC/Fernand-Nathan et Soc. Math. de France, Paris, 1981.

21. Grimaldi R., Pansu P. Sur la croissance du volume dans une classe conforme. J. Math. Pures Appl., (9) 71, No. 1, 1-19 (1992).

22. Гольдитейн В. М., Водопьлнов C. K. Метрическое пополнение области при помощи конформной емкости, инвариантное при квазиконформных отображениях. ДАН СССР, 238, №5, 1040-1042 (1978).

23. Hesse $J$. A $p$-extremal lenght and p-capacity equolity. Ark. Mat., 13, 131-144 (1975).

24. Hildebrandt $S$. Liouville theorems for harmonic mappings and approach to Bernstein theorems. Ann. Math. Stud., 102, 107-131 (1982).

25. Holopainen I. Nonlinear potencial theory and quasiregular mappings on riemannian manifolds. Ann. Acad. Sci. Fenn. Ser. AI, Math. Dissert., Vol. 74, Helsinki (1990).

26. Karp L. Subharmonic functions on real and complex manifolds. Math. Z., 178, 535-554 (1982). 
27. Кесельман В. М. О римановых многообразиях $p$-параболического типа. Изв. вузов, Математика, 4, 81-83 (1985).

28. Kilpelainen T. Potential Theory for Supersolutions of Degenerate Elliptic Equations. Indiana Univ. Math. J., 38, No. 2, 253-275 (1989).

29. Loewner $C$. On the conformal capacity in space. J. Math. Mech., 8, 411-414 (1959).

30. Milnor J. A note on curvature and fundamental group. J. Diff. Geom., 2, 1-7 (1968).

31. Milnor $J$. On desiding whether a surface is parabolic or hyperbolic. Amer. Math. Monthly, 84, No. 1, 43-46 (1977).

32. Мазья В. Г. Пространства С. Л. Соболева. Изд-во ЛГУ, Л., 1985.

33. Миклюков В. М. О конформном типе поверхностей, теореме Лиувилля и теореме Бернштейна. ДАН СССР, 242, No. 3, 537-540 (1978).

34. Мостов Г. Д. Квазиконформные отображения и жесткость гиперболических пространственных форм. Математика, сб. пер., 16, №5, 105-157 (1972).

35. Osserman R. Isoperimetric inequalities. Bull. Am. Math. Soc., 84, 1182-1238 (1978).

36. Pansu P. An isoperimetric inequality on the Heisenberg group. Proceedings of "Differential Geometry and Homogeneous Spaces", Torino, 1983, pp. 159-174.

37. Решетняк Ю. Г. Пространственные отображения с ограниченным искажением. Наука, Новосибирск, 1982.

38. Rickman S. Quasiregular mappings. Springer-Verlag, 1993.

39. Schlesinger E. Conformal Invariants and Prime Ends. Am. J. Math., 80, 83-102 (1958).

40. Sario L., Nakai M., Wang C., Chung L. O. Classification theory of Riemannian manifolds. Lecture Notes in Math., Vol. 605, Springer-Verlag, 1977.

41. Швари A. С. Объемный инвариант накрытий. ДАН СССР (НС), 105, 32-34 (1955).

42. Tanaka H. Harmonic boundaries of Riemannian manifolds. Nonlinear Analysis, Methods and Applications, 14, No. 1, 55-67 (1990).

43. Väisälä $J$. Lectures on $n$-dimensional quasiconformal mappings. Lecture Notes in Math., Vol. 229, Springer-Verlag, 1971.

44. Vuorinen $M$. Conformal geometry and quasiregular mappings. Lecture Notes in Math., Vol. 1319, Springer-Verlag, 1988.

45. Ziemer W. P. Extremal lenght as a capacity. Mich. Math. J., 17, 117-128 (1970).

46. Zorich $V$. A. On Gromov's geometric version of the global homeomorphism theorem for quasiconformal mappings. XIV Rolf Nevanlinna Colloquium, Helsinki, June 1014, 1990, Abstracts, p. 36.

47. Zorich $V$. A. The global homeomorphism theorem for space quasiconformal mappings, its development and related open problems. Lecture Notes in Math., Vol. 1508, $131-148,1992$.

Московский государственный университет, механико-математический факультет

Кемеровский государственный университет,

22 марта 1995 г.

математический факультет 